\title{
Revisão dos novos modelos de produção: Economia Circular, Bioeconomia e
}

\section{Biossociedade}

\author{
Review of the new production models: Circular Economy, Bioeconomy and Biosociety \\ Revisión de los nuevos modelos de producción: Economía Circular, Bioeconomía y Biosociedad
}

Recebido: 21/07/2021 | Revisado: 26/07/2021 | Aceito: 27/07/2021 | Publicado: 03/08/2021

\author{
Vitória dos Santos Fontanezi Maia \\ ORCID: https://orcid.org/0000-0003-1451-2990 \\ Universidade de São Paulo, Brasil \\ E-mail: fontanezi@usp.br \\ Antônio Emílio Shibata \\ ORCID: https://orcid.org/0000-0003-3063-7849 \\ Universidade de São Paulo, Brasil \\ E-mail: shibataae@usp.br \\ Ediene Monteiro Romão \\ ORCID: https://orcid.org/0000-0002-3413-0423 \\ Universidade de São Paulo, Brasi \\ E-mail: edieneromao@usp.br
}

\begin{abstract}
Resumo
O aumento populacional e a forma convencional de produção linear fazem com que seja necessário analisar conceitos referentes a economia circular, bioeconomia e biossociedade, teorias já existentes e que já são aplicáveis em muitos setores da sociedade. Nesse sentido, a preocupação com a qualidade de vida e do meio ambiente são precursores para o desenvolvimento de uma sociedade com um maior engajamento de novos modos de produção, mais limpos e otimizados. Diante disso, o presente artigo traz uma revisão bibliográfica sobre os novos modelos de produção, envolvendo a economia circular, a bioeconomia e a biossociedade. Tem como objetivo introduzir o conceito como uma alternativa tecnológica sustentável, que torna os processos mais limpos e econômicos, como a otimização de processos e aproveitamento de biomassa e outros resíduos. A pesquisa foi desenvolvida ao se construir uma revisão a respeito do tema, passando, então, por uma abordagem geral, apresentando a origem, aplicabilidade e características tecnológicas sobre a temática. Conclui-se, com ênfase, a importância de se estudar novos modelos de produção para promover e melhorar a capacidade de produção mais limpa e sustentável, além de potencializar a divulgação desses conceitos em meio a pesquisas.
\end{abstract}

Palavras-chave: Economia circular; Bioeconomia; Biossociedade; Sustentabilidade; Resíduos.

\begin{abstract}
Population increase and the conventional form of linear production make it necessary to analyze concepts related to circular economy, bioeconomy and biosociety, theories that already exist and that are already applicable in many sectors of society. In this sense, the concern with the quality of life and of the environment are precursors for the development of a society with a greater commitment to new, cleaner and more optimized modes of production. Therefore, this article brings a review on the new production models, involving the circular economy, bioeconomy and biosociety. Its objective is introducing the concept as a sustainable technological alternative, which makes processes cleaner and more economical, such as the optimization of processes and the use of biomass and other wastes. The research was developed by building a review on the topic, then going through a general approach, presenting the origin, applicability and technological characteristics of the topic. It concludes, with emphasis, the importance of studying new production models to promote and improve the capacity for cleaner and more sustainable production, in addition to enhancing the dissemination of these concepts through research.
\end{abstract}

Keywords: Circular economy; Bioeconomy; Biosociety; Sustainability; Wastes.

\section{Resumen}

El aumento poblacional y la forma convencional de producción lineal hacen necesario analizar conceptos relacionados con la economía circular, la bioeconomía y la biosociedad, teorías que ya existen y ya son aplicables en muchos sectores de la sociedad. En este sentido, la preocupación por la calidad de vida y el medio ambiente son precursores para el desarrollo de una sociedad con una mayor apuesta por modos de producción nuevos, más limpios y optimizados. Por tanto, este artículo trae una revisión bibliográfica sobre los nuevos modelos de producción, que involucran la economía circular, la bioeconomía y la biosociedad. Su objetivo es introducir el concepto como una alternativa tecnológica sostenible, que hace que los procesos sean más limpios y económicos, como la optimización de procesos y el uso de biomasa y otros residuos. La investigación se desarrolló construyendo una revisión sobre el tema, luego pasando por un enfoque general, presentando el origen, aplicabilidad y características tecnológicas del 
tema. Se concluye, con énfasis, la importancia de estudiar nuevos modelos productivos para promover y mejorar la capacidad de producción más limpia y sostenible, además de potenciar la difusión de estos conceptos a través de la investigación.

Palablas clave: Economía circular; Bioeconomía; Biosociedad; Sostenibilidad; Residuos.

\section{Introdução}

$\mathrm{O}$ crescimento populacional e o poder de consumo intensificam as complicações no que tange à demanda $\mathrm{e}$ disponibilidade dos recursos naturais e, consequentemente, ampliam os impactos socioambientais negativos.

Segundo a European Environment Agency (2016), com cerca de 7 bilhões de pessoas, o planeta já possui dificuldade para atender as demandas da população no que se refere em alimentação, moradia, além da extração de capital natural para produção de bens e serviços e a destinação de resíduos.

O cenário apresentado mostra-se insustentável para o futuro e, portanto, estudos iniciados no âmbito da sustentabilidade vêm ganhando força e estimulando iniciativas de gestão tendo o foco na harmonia entre meio ambiente e economia.

A sustentabilidade tão falada atualmente é base do desenvolvimento sustentável, no qual, os três elementos: econômico, social e ambiental, formam um conceito fundamental para a mudança do arquétipo já conhecido, o qual visa quase que exclusivamente o lucro.

Nesse contexto, a abordagem da Economia Circular (EC) é um divisor de águas em relação ao modelo convencional de produção; também chamado de modelo linear; uma vez que a EC se realizada desde o planejamento dos produtos, como elucidado por Oliveira (2018), possibilita a maximização do aproveitamento dos recursos e a minimização de possíveis residuais, aproximando as atividades antrópicas aos ciclos naturais

Visitando o foco ambiental, são observados, com uma frequência cada dia menos espaçada, o debate e o incentivo de transformações em sistemas de produção tradicionais para sistemas de gestão com viés sustentável. É necessária esta readequação com objetivo não só de cumprir às legislações impostas, como também encontrar a harmonia entre meio ambiente, sociedade e economia.

A implementação da gestão com viés sustentável abre portas para que as empresas possam utilizá-la como ferramenta de crescimento e vantagens competitivas dentro do mercado global (Leitão, 2015).

Nessa concepção, o presente trabalho apresenta uma narrativa voltada para a economia circular bem como seus princípios, benefícios e consequentemente abordar assuntos relacionados a bioeconomia e biossociedade.

\section{Metodologia}

A metodologia da pesquisa se destaca como exploratória e descritiva. Ela foi determinada por meio de levantamentos de pesquisas bibliográficas que, segundo Gil (2008), visam estudar um fenômeno que se deseja melhor compreender a fim de familiarizar-se com a problemática, construindo um maior número de hipóteses. Portanto, a pesquisa é descritiva, pois visa à descrição de características de certo grupo ou fenômeno. O embasamento teórico se pautou em dados e informações científicas levantadas em livros e artigos disponíveis na área de exploração.

Após a posse das informações, buscou-se fazer uma análise detalhada do objeto em estudo. Os resultados foram apresentados de modo qualitativo, abordados dessa forma por serem dados mais explicativos sobre o contexto da economia circular, bioeconomia e biossociedade, sendo obtidos por meio de análises e descrições da complexibilidade do tema proposto.

\section{Resultados e Discussão}

A seguir será apresentado o levantamento bibliográfico considerado relevante para embasar discussões sobre o tema. Portanto, os tópicos foram divididos em: Conceito-históricos; Modelos de produção; Economia e Sustentabilidade; Panorama 
Nacional e Internacional.

\subsection{Conceito-histórico}

Dentre os levantamentos bibliográficos no âmbito internacional, os conceitos de Economia Circular e Bioeconomia vêm obtendo posição de destaque em buscas de estratégias para o desenvolvimento sustentável (Wield et al., 2013).

\subsection{A economia circular}

Para Preston (2012), a EC apresenta uma visão, os quais os sistemas são abertos e os recursos são utilizados no ciclo de produção e a disposição final dos resíduos devem ser substituídos por sistemas que reutilizam e reciclam esses recursos. Já para a Ellen MacArthur Foundation (2015), a EC é uma economia que se regenera, guiada pelas energias renováveis e redução do desperdício, procurando reintroduzir materiais aos ciclos do produto, visando sua renovação.

A Ellen MacArthur Foundation (2015) foi criada para disseminar os conceitos sobre a EC de forma a orientar e implementar os três princípios estabelecidos para os convencionais modelos de produção, que são apresentados a seguir no Quadro 1.

Quadro 1- Princípios da Economia Circular

\begin{tabular}{|c|c|c|}
\hline & Princípios & Ações \\
\hline 1 & $\begin{array}{l}\text { Preservar e aprimorar o capital natural, } \\
\text { controlando } \\
\text { finitos e equilibrando os fluxos de } \\
\text { recursos renováveis. }\end{array}$ & $\begin{array}{l}\text { - Desmaterializar produtos e serviços, ou seja, realizar a entrega virtual, } \\
\text { sempre que possível; } \\
\text { - Selecionar recursos para produção priorizando os recursos renováveis; } \\
\text { - Buscar o aprimoramento do capital natural, capaz de criar condições } \\
\text { suficientes para a regeneração dos mais variados recursos naturais; }\end{array}$ \\
\hline 2 & $\begin{array}{l}\text { Otimizar o rendimento de recursos } \\
\text { fazendo } \\
\text { componentes e materiais no mais } \\
\text { alto nível de utilidade o tempo todo, } \\
\text { tanto no ciclo técnico quanto no } \\
\text { biológico. }\end{array}$ & $\begin{array}{l}\text { - Projetar novos produtos baseando-se na renovação e na reciclagem; } \\
\text { - Utilizar circuitos internos menores de para minimizar o consumo de } \\
\text { energia e preservar outros valores associados aos materiais; } \\
\text { - Estimular a inserção, de forma segura, de nutrientes biológicos na } \\
\text { biosfera para decomposição, com o objetivo de transformá-los em } \\
\text { matérias primas valiosas, em novos ciclos. }\end{array}$ \\
\hline 3 & $\begin{array}{llr}\text { Estimular a efetividade do sistema } \\
\text { revelando } & \text { e excluindo as } \\
\text { externalidades } & \text { negativas desde o } \\
\text { princípio } & & \\
\end{array}$ & $\begin{array}{l}\text { - Buscar a efetividade dos processos projetados, pois essa tende a reduzir } \\
\text { os danos de má utilização dos recursos e da má gestão. }\end{array}$ \\
\hline
\end{tabular}

Fonte: Adaptado de EMF (2015).

Os princípios elencados no Quadro 1 remetem à economia circular para desenvolvimento de atividades de produção em dois ciclos: o biológico e o técnico. No primeiro ciclo, encontram-se processos naturais que são habilitados a regenerar materiais tendo ou não intervenção humana, sendo assim, é um processo no qual parte do material será retornado a natureza. Já no ciclo técnico, juntando a intervenção humana a quantidade de energia suficiente, é possível a recuperação desses materiais e após a sua utilização podem se tornar matérias primas ao invés de resíduos (Gejer; Tennenbaum, 2017).

Portanto, é evidente a importância de quebrar paradigmas quanto ao modelo tradicional de produção, inserindo conceitos de economia circular e sustentabilidade nos debates, o qual, impulsiona-se a incorporar os conceitos de bioeconomia e biossociedade. 


\subsection{A bioeconomia}

O matemático e economista romeno Nicholas Georgescu-Roegen criou uma economia ecologicamente e socialmente sustentável, ao verificar questões econômicas integrando variáveis da biologia. Ele questionou que os recursos naturais tendem a ser degradados quando utilizados na atividade econômica e defendeu uma economia focada na ecologia (Georgescu-Roegen, 1971). Alguns pesquisadores consideram que os estudos de Georgescu-Roegen são precursores dos atuais conceitos da bioeconomia, cujo foco é mais voltado para o valor criado por novas atividades de negócios oriundas do uso inovador e sustentável dos recursos biológicos.

Segundo McCormick e Kautto (2013), ao definir a bioeconomia, há também a necessidade de se definir a biotecnologia, que pode ser considerada um conjunto de técnicas de processamento de insumos para sua transformação em produtos com conteúdo biológico.

Diante desses desafios, com longos prazos de solução, o progresso da bioeconomia demandará tantos avanços, que as políticas públicas têm um papel importante no seu desenvolvimento, tendo em vista seu potencial de geração de externalidades positivas, principalmente para um país com as características do Brasil, que possui grande potencial de produção de biomassa tanto vegetal e animal.

As empresas GranBio e Raízen são exemplos de empreendimentos voltados para o setor da bioeconomia. São responsáveis por construir usinas especializadas na produção de E2G (etanol de segunda geração, que é produzido do bagaço da cana-de-açúcar) em escala comercial. A Bioflex 1, usina industrial da GranBio localizada em São Miguel dos Campos (AL), foi a primeira fábrica de etanol celulósico no país com capacidade de produção de 82 milhões de litros do biocombustível por ano.

Além dessas duas iniciativas, destacam-se os empreendimentos das empresas Amyris (para fabricação de combustíveis e produtos derivados do farneseno utilizando como insumo a cana-de-açúcar), da TerraVia (antiga Solazyme, para produção de alimentos e especialidades químicas a partir de algas marinhas) e da Braskem (para produção de polietileno a partir da cana-de-açúcar).

\subsection{Biossociedade}

Em um mundo com recursos escassos, uma biossociedade é aquela que é sustentável e que mantém elementos valiosos em circulação, por uma série de novos usos e conversões entre seus ciclos de vida (Hobson; Lynch, 2016).

Existem alguns indicadores de sustentabilidade para uma biossociedade. Van de Kerk e Manuel (2008) desenvolveram um índice que engloba vários aspectos, o SSI (sigla em inglês para Índice de Sociedade Sustentável). É um parâmetro que leva em conta cinco categorias: "desenvolvimento pessoal", "ambiente limpo", "sociedade balanceada", "uso sustentável dos recursos" e "mundo sustentável". Dentre estas, são contemplados 22 indicadores, que vão desde "segurança sanitária” a "área florestal”. De acordo com os resultados da aplicação desse índice para 150 países, a Noruega foi determinada como o país mais sustentável. O Brasil ocupou a quinquagésima segunda posição no ranking (Van de Kerk; Manuel, 2008).

Como exemplos de outros índices que podem mensurar o grau de sustentabilidade de uma sociedade, tem se o "Índice de Desenvolvimento Humano", o "Índice de Progresso Genuíno" e os "Indicadores de Desenvolvimento do Milênio" (Van de Kerk; Manuel, 2008).

Na sociedade brasileira, uma aplicação da economia circular em prol da sustentabilidade pode ser observada na gestão e no gerenciamento de resíduos sólidos. A própria Política Nacional de Resíduos Sólidos, Lei no 12.305 de 2010, institui, através do artigo $9^{\circ}$, que: "Art. $9^{\circ} \mathrm{Na}$ gestão e gerenciamento de resíduos sólidos, deve ser obedecida a seguida ordem de prioridade: não geração, redução, reutilização, reciclagem, tratamento dos resíduos sólidos e disposição final ambientalmente adequada dos rejeitos" (BRASIL, 2010).

O artigo $3^{\circ}$ da mesma legislação dá certas definições de termos, dentre eles o de "reciclagem" e o de "reutilização". 
“Art. $3^{\circ} \mathrm{XIV} \mathrm{-} \mathrm{reciclagem:} \mathrm{processo} \mathrm{de} \mathrm{transformação} \mathrm{dos} \mathrm{resíduos} \mathrm{sólidos} \mathrm{que} \mathrm{envolve} \mathrm{a} \mathrm{alteração} \mathrm{de} \mathrm{suas} \mathrm{propriedades}$ físicas, físico-químicas ou biológicas, com vistas à transformação em insumos ou novos produtos [...]” (BRASIL, 2010). “Art $3^{\circ}$ XVIII - reutilização: processo de aproveitamento dos resíduos sólidos sem sua transformação biológica, física ou físicoquímica [...]" (BRASIL, 2010). Os objetivos da realização de tais ações é dar um novo uso a um material, ou então aproveitar alguns de seus elementos para a produção de novos.

No tratamento de alguns resíduos também é possível a aplicação de economia circular, ao converter determinada substância ou propriedade dos mesmos em energia. O inciso primeiro do Art. $9^{\circ}$ faz uma menção não direta sobre possibilidades no tratamento: “Art $9^{\circ} \S 1^{\circ}$ Poderão ser utilizadas tecnologias visando à recuperação energética dos resíduos sólidos urbanos, desde que tenha sido comprovada sua viabilidade técnica e ambiental e com a implantação de programa de monitoramento de emissão de gases tóxicos aprovados pelo órgão ambiental” (BRASIL, 2010).

Infelizmente, segundo estimativa da Associação Brasileira de Empresas de Limpeza Pública e Resíduos Especiais (ABRELPE), feita em 2018, a porcentagem de reciclagem dos resíduos sólidos no Brasil é de apenas 3\% (Marasciulo, 2020).

\subsection{Modelos de produção}

Com a revolução industrial, os processos de produção foram configurados de modo linear, no qual, eram extraídos os recursos naturais e a partir destes eram produzidos um bem material, em seguida, após uso ou consumo eram descartados incorretamente no meio ambiente. Além disso, inclui-se também, os resíduos gerados durante o processo e sua disposição inadequada (Azevedo, 2020). Esse processo é ilustrado pela Figura 3.

Figura 1 - Fluxo do modelo de economia linear

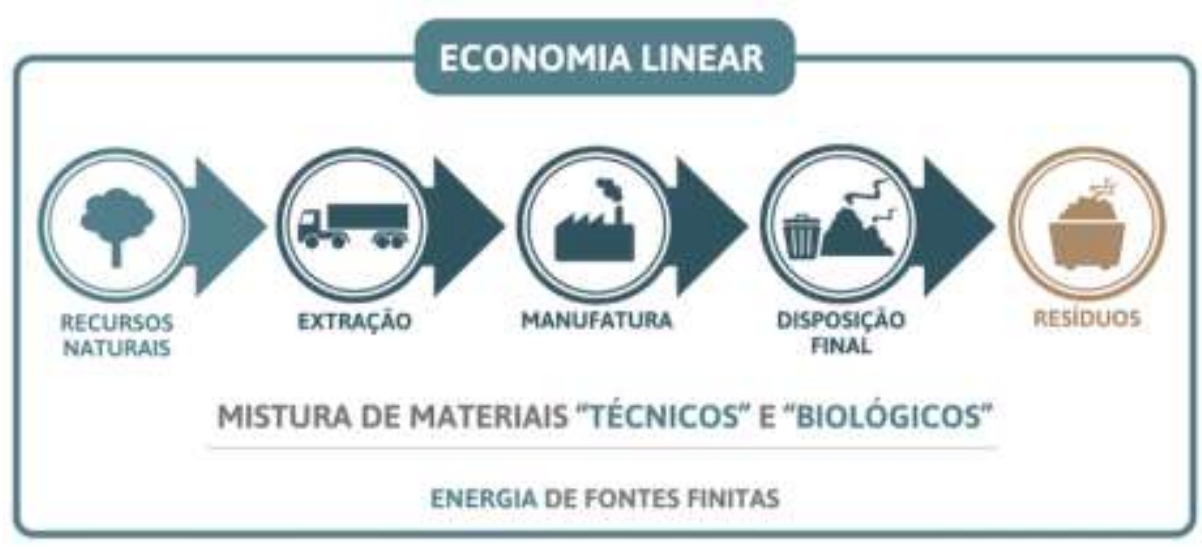

Fonte: Spallicci, 2018.

Michelini et al. (2017) afirmam a existência de um consenso sobre o atual cenário onde há um grave esgotamento de recursos naturais e o sistema socioeconômico linear, caracterizado pelo descarte do produto em seu final de vida útil.

O conceito fundamental da economia circular é baseado em eliminar o desperdício. As ações referentes à EC não são limitadas apenas aos processos industriais, mas aos processos de serviço e diferentes modelos de negócios (Azevedo, 2020). Esse processo é ilustrado na Figura 4 a seguir. 
Figura 2 - Fluxo do modelo de Economia Circular

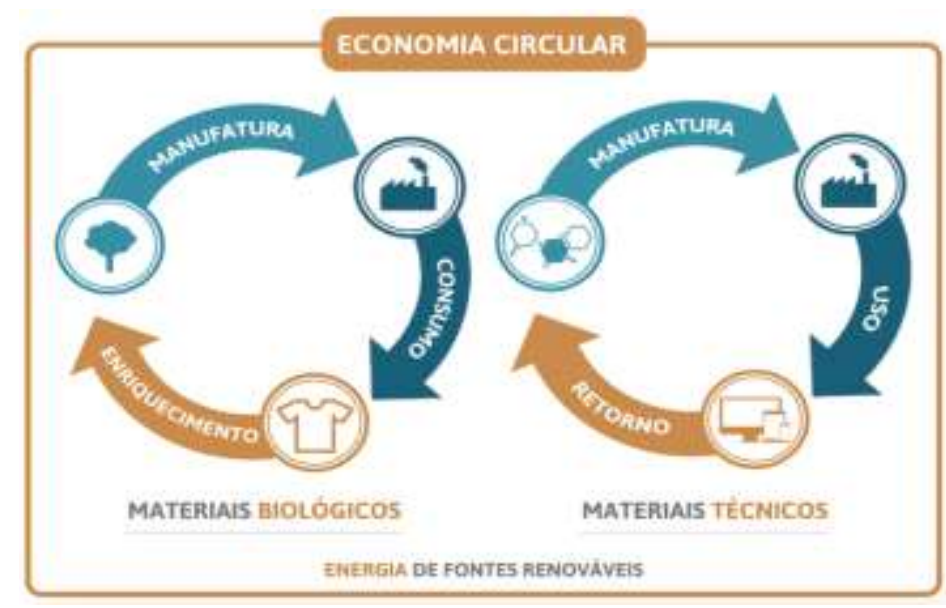

Fonte: Spallicci, 2018.

Recentemente, emergiu-se uma pressão para estimular a transição da atual sociedade para uma mais sustentável tendo como premissa a mitigação de problemas ambientais como a redução da biodiversidade, exploração exacerbada de recursos e a poluição ambiental como um todo (Martin et al, 2017).

\subsection{Panoramas Local e Internacional: Um comparativo}

Nesse tópico serão apresentados os panoramas a nível nacional e internacional respectivamente visando obter dados gerais e realizar um comparativo da temática nesses dois campos. O panorama possui a intenção de dar subsídio as citações e ampliar a visão sobre a economia circular e sua disseminação pelo globo.

\subsubsection{Panorama Nacional}

No Brasil e na América do Sul, os avanços da economia circular na política ainda estão na fase inicial (Abdalla \& Sampaio, 2018).

O maior apoio se dá por parte das universidades e suas pesquisas. A Universidade de São Paulo, por exemplo, desde 2016, integra o Pioneer Universities, um grupo composto por mais seis instituições acadêmicas da Europa e dos Estados Unidos. O acordo entre elas é de disseminar e desenvolver um modelo sistêmico de economia circular (Abdalla \& Sampaio, 2018).

Uma iniciativa para a Américas foi a criação da Plataforma de Economia Circular das Américas (PEC-Américas), que é um site com informações sobre o andamento da economia circular nesses continentes (Abdalla; Sampaio, 2018).

Por iniciativa desse site, foi realizado em 2017, na Colômbia, o "Circular Economy Forum of the Americas", um evento de grande repercussão para o público especializado. A exemplo, no Uruguai foi realizado o "Fórum de Economia Circular" (Abdalla \& Sampaio, 2018).

O Chile é pioneiro em ações efetivas, ao criar o "Centro de Inovación y Economía Circular", em 2016. Este projeto incentiva ideias inovadoras, ao fornecer orientação em laboratório e desenvolvimento de protótipos (Abdalla \& Sampaio, 2018).

\subsubsection{Panorama Internacional}

Fitch-Roy et al (2021) pesquisaram políticas otimizadas de economia circular em 60 países, focando na área de aproveitamento de resíduos, através de dados documentados dos governos e de trabalhos acadêmicos. Analisaram de acordo 
com um modelo de aplicação ótima proposto através de estudo. Essa pesquisa foi feita entre 2018 e 2019, e teve como resultado que dos 60 países verificados, 10 apresentavam uma completa integração entre economia circular e gerenciamento dos resíduos; 21 possuíam uma forte integração; 22 tinham uma fraca relação; e 7 não recuperavam recursos.

Os autores também citaram alguns documentos implementados na política de alguns países, como o "Promoção de Economia Circular" adotado em 2008 (alterado em 2018) na China; o "Liderando o Ciclo: o Guia Finlandês para uma Economia Circular 2016-2025" implementado em 2016 na Finlândia; o "Guia para a Economia Circular na Eslovênia" aderido em 2018, pela Eslovênia; dentre outros.

Aproveitando o exemplo sobre resíduos sólidos mencionado na seção sobre biossociedade, e o dado da ABRELPE que revela que no Brasil a porcentagem de reciclagem é de apenas 3\%, compara-se com outros países do mundo. A Alemanha é o país com a maior porcentagem de reciclagem dos resíduos sólidos, atingindo 66,7\%, conforme dados da EuroStat (2019). A proporção média de reciclagem na União Europeia, em 2019, é de 47,7\%; contendo países como Itália (51,9\%), Espanha $(34,7 \%)$, Portugal $(28,9 \%)$ e outros. Dos 20 países da União Europeia que possuíam esses dados disponíveis pela EuroStat, apenas 4 possuíam uma taxa de reciclagem menor que 20\%: Chipre (15,0\%), Romênia (11,5\%), Turquia (11,5\%) e Malta $(8,9 \%)$; todos, portanto, acima da porcentagem brasileira.

Segundo um dado publicado em 2018 pela ONU Meio Ambiente (2021), na América Latina e no Caribe, 90\% dos resíduos não são reaproveitados. Apesar da economia circular poder ser aplicada nas mais diversas áreas de produção e consumo, os dados de resíduos sólidos são os mais disponíveis, por fazerem parte de legislações dos países, e por isso foram os apresentados no presente trabalho.

A União Europeia criou, em 2015, o Acordo Verde Europeu para Crescimento Sustentável (European Green Deal for Sustainable Growth). Contido nele, um dos setores é o Plano de Ação de Economia Circular. O texto do documento retrata as necessidades consequentes da economia linear utilizada até então, e esclarece que se leva em torno de vinte e cinco anos para transformar um setor industrial. O documento, portanto, direciona essa implementação da economia circular na Europa (European Commission, 2019).

A Europa foi a pioneira para a realização de mudanças em seus processos produtivos. É interessante o feito realizado em uma cidade da Dinamarca, que em 1980 promoveu uma "simbiose industrial", isto é, formam um parque industrial onde uma indústria se instala nas vizinhanças de outra para aproveitar seus resíduos (Abdalla \& Sampaio, 2018).

\section{Conclusão}

O presente trabalho procurou definir os conceitos de Economia Circular, Bioeconomia e Biossociedade abordando seus princípios, benefícios e aplicações por intermédio de um levantamento bibliográfico considerado relevante para embasar o tema.

Através dos elementos abordados, o estudo permitiu avançar na disseminação do tema que tem sido norteadora de ações de diversos países e aponta-se a necessidade de maiores esforços para inserir a Economia Circular no sistema de produção brasileiro tendo como premissa que o modelo convencional de produção é insustentável em virtude de seus inúmeros impactos socioambientais negativos o que, consequentemente, influenciará na qualidade de vida de novas gerações.

Visa-se que a Bioeconomia e a Economia Circular podem atuar na mitigação de impactos negativos tanto em âmbito ambiental quanto social e econômico, uma vez que, o meio ambiente está intrínseco às relações do homem e o modelo de produção de bens e serviços. Sugere-se para a realização de trabalhos futuros fazer um levantamento de empresas que praticam economia circular ou bioeconomia no setor nacional. 


\section{Referências}

Abdalla, F. A., \& Sampaio, A. C. F. (2018). Os novos princípios e conceitos inovadores da Economia Circular. Entorno Geográfico, (15), 82-103.

Azevedo, F. P. M. D. (2020). A educação ambiental como vetor para fomentar a economia circular: um estudo de caso em uma escola pública da cidade de Manaus.

BRASIL. Lei $\mathrm{n}^{\circ}$ 12.305, de 02 de agosto de 2010. Institui a Política Nacional de Resíduos Sólidos; altera a Lei nº 9.605 , de 12 de fevereiro de 1998 ; e dá outras providências.<http://www.planalto.gov.br/ccivil_03/_ato2007-2010/2010/lei/112305.htm>.

Comissão Europeia. Strategy for "Innovating for sustainable grow: a bioeconomy for Europe". <https://ec.europa.eu/research/bioeconomy/pdf/officialstrategy_en.pdf>.

Ellen MacArthur Foundation. (2015). Delivering the circular economy: A toolkit for policymakers. Ellen MacArthur Foundation.

EUROPEAN COMMISSION. The European Green Deal. <https://ec.europa.eu/info/sites/info/files/european-green-deal-communication_en.pdf>.

EUROSTAT. Recycling Rate of Municipal Waste. 〈https://ec.europa.eu/eurostat/databrowser/view/t2020_rt120/default/table?lang=en>.

Fitch-Roy, O., Benson, D., \& Monciardini, D. (2021). All around the world: Assessing optimality in comparative circular economy policy packages. Journal of Cleaner Production, 286, 125493.

Geger, L., \& Tennenbaum, C. (2017). Os Três Princípios do Design Circular: Cradle to Cradle. Ideia Circular.

Georgescu-Roegen, N. (1971). The Entropy Lawandthe Economic Process Cambridge 1971. Georgescu-RoegenThe Entropy Law and the Economic Process 1971 .

Gil, A. C. (2008). Métodos e técnicas de pesquisa social. 6. ed. Ediitora Atlas SA.

Hobson, K., \& Lynch, N. (2016). Diversifying and de-growing the circular economy: Radical social transformation in a resource-scarce world. Futures, 82, $15-25$.

Leitão, A. (2015). Economia circular: uma nova filosofia de gestão para o séc. XXI. Portuguese Journal of Finance, Management and Accounting, 1(2), 150171

Marasciulo, M. Por que o Brasil ainda recicla tão pouco (e produz tanto lixo)? < https://revistagalileu.globo.com/Ciencia/Meio-Ambiente/noticia/2020/02/porque-o-brasil-ainda-recicla-tao-pouco-e-produz-tanto-lixo.html>.

Martin, G., Savaget, P., Bocken, N. M., \& Jan Hultink, E. (2017). The circular economy-A new sustainability paradigm. J. Clean. Prod, 423, 757-768.

McCormick, K.; Kautto, N.(2013). The Bioeconomy in Europe: an overview. Sustainability,. 5, n. 6, p. 2.589-2.608.

Michelini, G., Moraes, R. N., Cunha, R. N., Costa, J. M., \& Ometto, A. R. (2017). From linear to circular economy: PSS conducting the transition. Procedia CIRP, 64, 2-6.

OECD. Publishing. (2009). The Bioeconomy to 2030: Designing a policy agenda. Organisation for Economic Co-operation and Development.

Oliveira, F. R. Estratégias de economia circular: do desenvolvimento de produtos em arranjos produtivos locais às experiências internacionais. (2018) 138 f. Tese (Doutorado em Sistema de Gestão Sustentáveis) Universidade Federal Fluminense, Niterói, 2018.

Organização das Nações Unidas (ONU). World population to hit 9.8 billion by 2050, despite nearly universal lower fertility rates - UN. <https://refugeesmigrants.un.org/world-population-hit-98-billion-2050-despite-nearly-universal-lower-fertility-rates-\%E2\%80\%93-un>.

Preston, F. (2012). A global redesign? Shaping the circular economy.

Van de Kerk, G., \& Manuel, A. R. (2008). A comprehensive index for a sustainable society: The SSI- The Sustainable Society Index. Ecological Economics, $66(2-3), 228-242$.

Wield, D., Hanlin, R., Mittra, J., \& Smith, J. (2013). Twenty-first century bioeconomy: Global challenges of biological knowledge for health and agriculture. Science and Public Policy, 40(1), 17-24. 